\title{
Risk Factors for Brain Damage in Preterm Infants After Late-Onset Circulatory Collapse Events
}

\author{
Eun Sun Lee ${ }^{1}$, Jin A Sohn ${ }^{2}$, Han-Suk Kim ${ }^{1}$, Ju Sun $\mathrm{Heo}^{3}$, and Jin A Lee ${ }^{2}$ \\ ${ }^{1}$ Department of Pediatrics, Seoul National University Children's Hospital, Seoul National University College of Medicine, Seoul, Korea \\ ${ }^{2}$ Department of Pediatrics, Seoul Metropolitan Government Seoul National University Boramae Medical Center, Seoul National \\ University College of Medicine, Seoul, Korea \\ ${ }^{3}$ Department of Pediatrics, Korea University Anam Hospital, Korea University College of Medicine, Seoul, Korea
}

\section{ABSTRACT}

Purpose: This study aimed to identify risk factors for brain damage in infants with late-onset circulatory collapse (LCC), a circulatory failure that responds to glucocorticoid therapy.

Methods: We retrospectively reviewed 167 infants (gestational age $<35$ weeks) who had hypotension between April 2009 and March 2017 at Boramae Medical Center. Forty infants were diagnosed with LCC and divided into two groups based on ultrasonography and magnetic resonance imaging findings: infants with periventricular leukomalacia ( $\mathrm{n}=9$ ) and those with normal images (n=31) after LCC. The clinical factors of these two groups, including perinatal characteristics, clinical features during the LCC period, and neonatal morbidities, were compared.

Results: There were no significant differences in perinatal characteristics and postnatal morbidities between the two groups. Postnatal age was greater in the group with brain damage ( 16 days vs. 24 days, $P=0.047$ ). The lowest mean blood pressure (MBP) and lowest serum sodium concentration were significantly lower in the brain damage group (19 mm Hg vs. $22 \mathrm{~mm} \mathrm{Hg}, P=0.034 ; 125 \mathrm{mmol} / \mathrm{L}$ vs. $129 \mathrm{mmol} / \mathrm{L}, P=0.043$ ). There were no significant differences in other clinical factors, including cortisol levels, and inotrope and hydrocortisone use. In multivariate logistic regression, older postnatal age (odds ratio [OR], 1.147; $P=0.049$ ), lower MBP (OR, 0.616; $P=0.031$ ), and lower sodium concentration (OR, $0.728 ; P=0.037$ ) during the LCC period highly predicted brain damage in infants with LCC (area under the curve $0.882, P=0.001$ ).

Conclusion: Close monitoring of LCC signs even in long-term stable preterm infants and management for preventing severe hyponatremia and hypotension are important to minimize the occurrence of brain damage in infants with LCC.

Key Words: Adrenal insufficiency; Leukomalacia, periventricular; Infant, premature
Received: 30 September 2018

Revised: 2 November 2018

Accepted: 29 November 2018

Correspondence to: Jin A Sohn

Department of Pediatrics, Seoul Metropolitan Government Seoul National University Boramae Medical Center, Seoul National University College of Medicine, 20 Boramae-ro 5-gil, Dongjak-gu, Seoul 07061, Korea

Tel: +82-2-870-2367

Fax: +82-2-831-0714

E-mail: jinasohn@hanmail.net https://orcid.org/0000-0002-4992-7128
Copyright(c)

By Korean Society of Neonatology.

All right reserved.

This is an Open-Access article distributed under the terms of the Creative Commons Attribution Non-Commercial License (http://creativecommons.org/licenses/ by-nc/4.0), which permits unrestricted non-commercial use, distribution, and reproduction in any medium, provided the original work is properly cited. 


\section{INTRODUCTION}

Hypotension is common in preterm infants, and maintaining organ flow through active management is critical in this condition. There are several reasons for hypotension, including hypovolemia, cardiac dysfunction, and sepsis-induced vasodilation. In addition, preterm infants can develop refractory hypotension that is resistant to volume expanders and inotropes and responds only to glucocorticoid therapy. This is explained by maladaptation of transient adrenocortical insufficiency during prematurity

${ }^{1)}$. Usually, hypotension occurs within 7 days; however, late-onset refractory hypotension has been reported in Japan since 2000 and is called late-onset circulatory collapse (LCC) ${ }^{2)}$. A Japanese review reported the incidence of LCC to be $6.3 \%$ in very low birth weight infants and $11.6 \%$ in extremely low birth weight infants ${ }^{3)}$. LCC may develop more frequently in smaller infants.

The hemodynamic condition associated with LCC is similar to the distributive shock pattern in sepsis. The ejection fraction increases and afterload decreases during the LCC period. The mean blood flow velocity increases in the superior mesenteric artery, whereas it decreases in the anterior cerebral and renal arteries ${ }^{4)}$. Because of this hemodynamic nature of LCC, cerebral blood flow to the white matter decreases and periventricular leukomalacia (PVL) can develop in patients ${ }^{5}$. In a previous report, although LCC did not cause brain damage in all cases, the neurologic prognosis was worse in severe cases ${ }^{6}$. Nakanishi et al. ${ }^{7)}$ reported that significantly more infants with LCC than controls had cerebral palsy at 3 years of age. However, no study has investigated the clinical characteristics that cause brain damage in infants with LCC. Therefore, the aim of this study was to compare the characteristics between infants with brain damage and normal infants after LCC events, and to identify the risk factors for brain damage in infants with LCC.

\section{MATERIALS AND METHODS}

\section{Subjects}

We extracted the data of infants with hypotensive events from among inborn preterm infants with gestational age (GA) of $<35$ weeks, who were admitted to the neonatal intensive care unit (NICU) of Seoul Metropolitan Government Seoul National University Boramae Medical Center from April 2009 to March 2017. We excluded infants with early-onset hypotension (within 7 days), cardiac diseases, confirmed infection, necrotizing enterocolitis (NEC), massive hemorrhage, pneumothorax, and congenital anomaly. We included preterm infants meeting the LCC criteria reported in a previous study ${ }^{3}$.

\section{Trial design}

We retrospectively reviewed electronic medical records of infants with LCC and divided them into two groups based on ultrasonography and magnetic resonance imaging (MRI) findings. We compared the brain images obtained before and after LCC events, and infants with white matter changes were classified into the brain lesion group. White matter changes included white matter injury, PVL, and macrocystic encephalomalacia. Ultrasonography examination was performed by one pediatric radiologist using iU22 (Phillips, Bothell, WA, USA) with a 5- to 8-Hz sector and a 5- to 12-MHz linear transducer. The ultrasonography and MRI findings were reviewed by two radiologists.

We compared and analyzed the perinatal clinical characteristics, clinical and laboratory factors during the LCC period, and neonatal complications between the brain lesion and normal brain groups. The LCC period was defined as the time from the diagnosis of LCC to the improvement of hypotension and oliguria without medication.

This study was approved by the Institutional Review Board of Boramae Medical Center, which waived the requirement for informed consent (approval no. 20-2017-42). This retrospective case-control study was conducted in compliance with the current revision of the Declaration of Helsinki and Good Clinical Practice guidelines.

\section{Definition of diseases}

LCC was diagnosed according to the clinical criteria suggested by the Japanese Study Groups for Neonatal Endocrinology based on a study by Koyama et al. ${ }^{3)}$ These criteria were as follows: (1) LCC occurring outside the transitional period (>7 days); (2) stable period before the onset of LCC; (3) no apparent causes such as sepsis, massive bleeding, or NEC before LCC onset; (4) hypotension and/or oliguria with sudden onset; and (5) hypotension and/or oliguria resistant to intravenous volume expanders and inotropes.

Hydrocortisone treatment was initiated after diagnosis according to the LCC criteria. We administered $1 \mathrm{mg} / \mathrm{kg}$ hydrocortisone four times every 6 hours. When symptoms improved, tapering was performed at $0.5 \mathrm{mg} / \mathrm{kg}$ every 6 hours for 2 days and $0.3 \mathrm{mg} /$ 
kg every 8 hours for 2 days $^{8)}$. The tapering schedules were modified according to the patients' recovery status from hypotension and the need for inotropes. Hypotension was defined as a mean blood pressure (MBP) of $<30 \mathrm{~mm} \mathrm{Hg}$ or a $15 \%$ reduction in systemic pressure ${ }^{9,10)}$. We checked the blood pressure using a noninvasive blood pressure monitoring device (Solar 8000i, GE Medical Systems Information Technologies, Milwaukee, WI, USA). A decrease in urine output of $>50 \%$ or a urine output of $<1 \mathrm{~mL} / \mathrm{kg} / \mathrm{hr}$

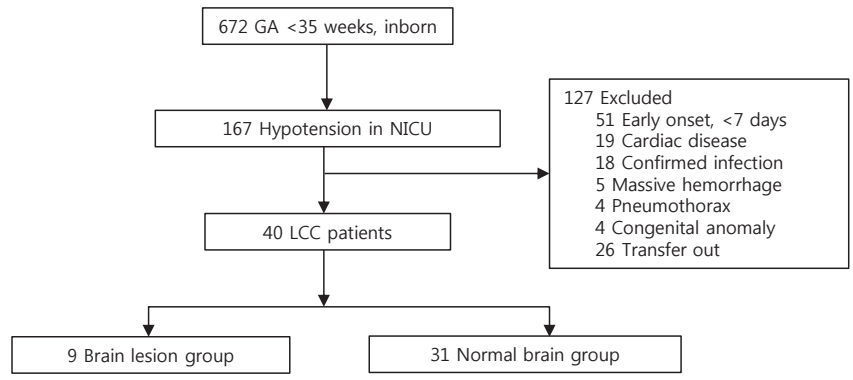

Figure 1. Flow diagram of the study. Brain lesion was defined as the presence of a periventricular white matter injury in ultrasonography and/or magnetic resonance images after late-onset circulatory collapse events. Abbreviations: GA, gestational age; NICU, neonatal intensive care unit; LCC, late-onset circulatory collapse. for 6 hours was defined as oliguria ${ }^{2)}$.

\section{Statistical analysis}

Statistical analyses were performed using the SPSS Statistics version 20.0 software (IBM Co., Armonk, NY, USA). Data were summarized as median with ranges or numbers with percentages. Qualitative data were analyzed using the chi-square test, and quantitative data were analyzed using the Mann-Whitney $U$-test to compare the two groups. Multivariate logistic regression was used for risk factor analysis. We also used receiver operating characteristic (ROC) curves to obtain cutoff values of significant factors. The cutoff point for parameters was defined as the point on the curve where the sum of sensitivity and specificity was the highest. $P$-values $<0.05$ were considered statistically significant.

\section{RESULTS}

\section{Study population}

Of 672 preterm infants ( $<35$ weeks of gestation) admitted to the NICU, 167 infants had hypotension. We excluded 127 infants
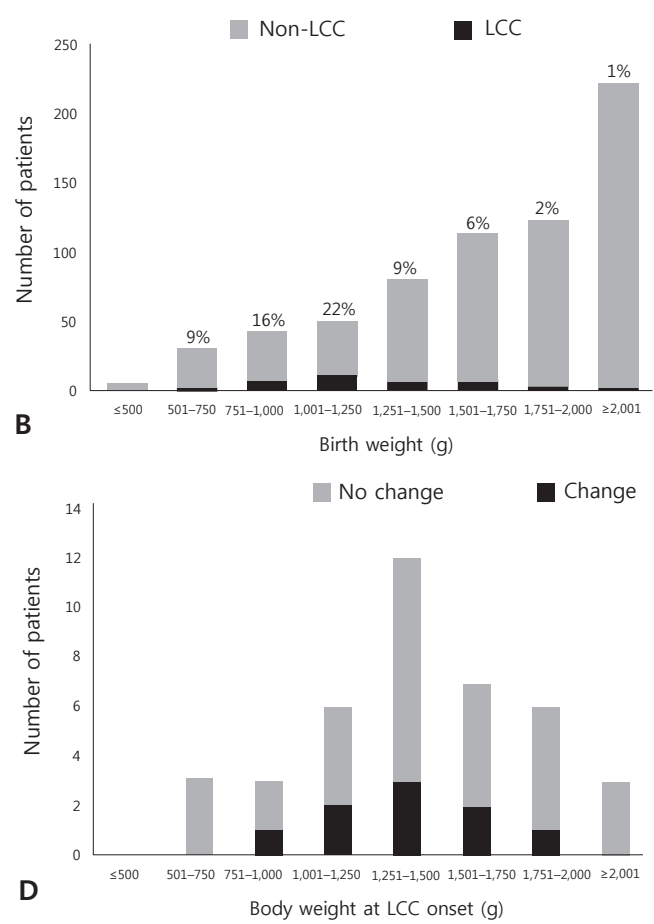

Figure 2. Distribution of late-onset circulatory collapse (LCC) and brain damage according to age and weight. (A) Distribution of LCC according to gestational age. (B) Distribution of LCC according to birth weight. (C) Distribution of brain damage in infants with LCC according to postmenstrual age at LCC onset. (D) Distribution of brain damage in infants with LCC according to body weight at LCC onset. 
who had hypotension within 7 days or with other causes of hypotension. We extracted the data of 40 infants identified according to the LCC criteria. There were nine infants in the brain lesion group and 31 infants in the normal brain group (Figure 1).

\section{Incidence and distribution of LCC}

In this study, the incidence of the LCC was $6 \%(n=40)$. The distribution showed that LCC had the highest frequency of occurrence in infants with a GA of 26 weeks (29\%) and in those with a birth weight of 1,001 to $1,250 \mathrm{~g}(22 \%)$. The incidence of brain damage in infants with LCC was $23 \%(n=9)$. The distribution showed that brain damage had the highest frequency of occurrence in infants with LCC with a postmenstrual age (PMA) of 30 weeks (50\%) and body weight of 751 to 1,250 g (33\%) (Figure 2).

\section{Perinatal clinical characteristics and neonatal complications}

The median GA and body weight at birth of infants with LCC were $29^{+6}$ weeks and $1.21 \mathrm{~kg}$, respectively. There were no significant differences in perinatal characteristics such as GA, birth weight, sex, multiple births, Apgar score, and delivery mode between the two groups (Table 1). Among maternal factors, there were no differences in premature rupture of membrane, chorio- amnionitis, preeclampsia, and antenatal steroid use (Table 1). There were no significant differences in neonatal complications such as respiratory distress syndrome, bronchopulmonary dysplasia, patent ductus arteriosus, NEC, retinopathy of prematurity (ROP), and Synthroid medication between the two groups (Table 1). However, the incidences of ROP stage III or higher (22.2\% vs. $3.2 \%, P=0.057)$ and thyroid hormone supplementation (33.3\% vs. $3 \%, P=0.080$ ) were marginally greater in the brain lesion group.

\section{Clinical and laboratory factors at the LCC period}

Postnatal age (PNA) was greater in the group with brain damage ( 16 days vs. 24 days, $P=0.047$ ). The PMA and body weight at LCC onset were not significantly different. The lowest MBP was significantly lower in the brain damage group $(19 \mathrm{~mm} \mathrm{Hg}$ vs. $22 \mathrm{~mm} \mathrm{Hg}, P=0.034$ ); however, the hypotension duration was not significantly different. The presence of oliguria, serum blood urine nitrogen level, and creatinine level were not different between the two groups. However, $85 \%$ of infants with LCC showed increased serum creatinine level. Among all infants with LCC, most showed stable respiration with room air (37.5\%) and needed minimal respiratory support (57.5\%). There was no difference in the need for respiratory support before and after LCC events

Table 1. Perinatal Characteristics and Neonatal Complications

\begin{tabular}{|c|c|c|c|c|}
\hline Characteristic & Total $(n=40)$ & Normal $(n=31)$ & Brain lesion $(n=9)$ & $P$-value \\
\hline Gestational age (wk) & $29^{+6}\left(25^{+0}-32^{+3}\right)$ & $29^{+6}\left(25^{+0}-32^{+3}\right)$ & $29^{+1}\left(26^{+1}-31^{+2}\right)$ & 0.702 \\
\hline Birth weight $(\mathrm{kg})$ & $1.21(0.54-1.82)$ & $1.25(0.54-1.82)$ & $1.20(0.85-1.64)$ & 0.545 \\
\hline Sex, male:female & $19(47.5): 21(52.5)$ & $16(51.6): 15(48.4)$ & $3(33.3): 6(66.7)$ & 0.334 \\
\hline Multiple births & $18(45.0)$ & $12(38.7)$ & $6(66.7)$ & 0.138 \\
\hline \multicolumn{5}{|l|}{ Apgar score } \\
\hline At 1 min & $5.5(1-8)$ & $5(1-8)$ & $6(2-7)$ & 0.949 \\
\hline At 5 min & $7(2-10)$ & $7(2-10)$ & $7(5-8)$ & 0.503 \\
\hline Caesarean section & $27(67.5)$ & $22(71.0)$ & $5(55.6)$ & 0.385 \\
\hline PROM & $24(60.0)$ & $20(64.5)$ & $4(44.4)$ & 0.279 \\
\hline Chorioamnionitis & $21(52.5)$ & $17(54.8)$ & $4(44.4)$ & 0.583 \\
\hline Preeclampsia & $3(7.5)$ & $3(9.7)$ & 0 & 0.332 \\
\hline Received antenatal steroid (0:1:2 dose) & $5(12.5): 15(37.5): 20(50.0)$ & $4(12.9): 11(35.5): 16(51.6)$ & 1 (11.1):4 (44.4):4 (44.4) & 0.887 \\
\hline RDS, surfactant & $14(35.0)$ & $10(32.3)$ & $4(44.4)$ & 0.500 \\
\hline $\mathrm{BPD}, \geq$ moderate & $6(15.0)$ & $5(16.1)$ & $1(11.1)$ & 0.711 \\
\hline PDA, treatment & $17(42.5)$ & $13(41.9)$ & $4(44.4)$ & 0.893 \\
\hline NEC, $\geq$ stage II & $2(5)$ & $2(6.5)$ & 0 & 0.434 \\
\hline ROP, $\geq$ stage III & $3(7.5)$ & $1(3.2)$ & $2(22.2)$ & 0.057 \\
\hline T4 medication & $6(15.0)$ & $3(9.7)$ & $3(33.3)$ & 0.080 \\
\hline
\end{tabular}

Values are expressed as median (range) or number (\%).

Abbreviations: PROM, premature rupture of membrane; RDS, respiratory distress syndrome; BPD, bronchopulmonary dysplasia; PDA, patent ductus arteriosus; NEC, necrotizing enterocolitis; ROP, retinopathy of prematurity; T4 medication, synthyroid treatment. 
between the two groups. The lowest serum sodium level was significantly lower in the brain damage group (125 mmol/L vs. $129 \mathrm{mmol} / \mathrm{L}, P=0.043)$; however, serum potassium and cortisol levels were not significantly different. There were no significant differences in inotrope and hydrocortisone use, hydrocortisone initiation time, and treatment duration between the two groups (Table 2).

\section{Risk factor analysis}

In multivariate logistic regression analysis, PNA (odds ratio [OR], 1.147; 95\% confidence interval [CI], 1.000 to $1.315 ; P=0.049)$, lowest MBP (OR, 0.616; 95\% CI, 0.397 to $0.958 ; P=0.031$ ), and lowest sodium level (OR, $0.728 ; 95 \% \mathrm{CI}, 0.540$ to $0.981 ; P=0.037$ ) were significantly associated with brain damage in infants with LCC ( $P=0.001, R^{2}=0.494$ ) (adjusted for oliguria, ROP, and thyroid hormone medications with borderline significance; $P<0.1$ ).

The ROC curves for predictors of brain damage in infants with LCC are shown in Figure 3. The cutoff values of PNA, MBP, and serum sodium level were 20.0 days (sensitivity=0.556, specificity=
0.677), $20.5 \mathrm{~mm} \mathrm{Hg}$ (sensitivity=0.667, specificity=0.581), and $127.5 \mathrm{mmol} / \mathrm{L}$ (sensitivity=0.667, specificity=0.677), respectively. The area under the ROC curve for the predicted probability of our logistic regression model was 0.882 (95\% CI, 0.771 to 0.992 ; $P=0.001$ ), which was more predictive of brain damage in infants with LCC than each risk factor.

\section{DISCUSSION}

A few studies have investigated the treatment of LCC in the past. Since the LCC concept was introduced in the 2000s, no treatment protocol has been established yet. Kobayashi et al. ${ }^{11}$ reported that LCC is significantly associated with cystic PVL, especially late-onset PVL. In addition, LCC has an important influence on neurologic prognosis, such as the development of cerebral palsy $^{7}$. To prevent brain damage in infants with LCC, it is important to understand the clinical features and factors that affect brain damage in this group. In this study, we analyzed 40

Table 2. Clinical and Laboratory Factors during the Late-Onset Circulatory Collapse Period

\begin{tabular}{|c|c|c|c|c|}
\hline Variable & Total $(n=40)$ & Normal $(n=31)$ & Brain lesion $(n=9)$ & $P$-value \\
\hline Postnatal age (d) & $18.5(7-44)$ & $16.0(7-44)$ & $24.0(15-35)$ & 0.047 \\
\hline Postmenstrual age (wk) & $32^{+1}\left(26^{+5}-36^{+2}\right)$ & $32^{+1}\left(26^{+5}-36^{+2}\right)$ & $32^{+4}\left(30^{+3}-34^{+4}\right)$ & 0.924 \\
\hline Bodyweight (kg) & $1.43(0.70-2.30)$ & $1.46(0.70-2.30)$ & $1.33(0.90-1.80)$ & 0.610 \\
\hline Hypotension duration (d) & $3(1-18)$ & $3(1-15)$ & $5(1-18)$ & 0.849 \\
\hline Lowest MBP (mm Hg) & $21(15-31)$ & $22(15-31)$ & $19(15-22)$ & 0.028 \\
\hline Oliguria $(\mathrm{mL} / \mathrm{kg} / \mathrm{hr})$ & $1.25(0.00-4.20)$ & $1.50(0.00-4.20)$ & $0.80(0.30-2.09)$ & 0.092 \\
\hline Respiratory support needed just before event* & $\begin{array}{c}15: 3: 20: 2 \\
(37.5: 7.5: 50.0: 5.0)\end{array}$ & $\begin{array}{c}12: 2: 15: 2 \\
(38.7: 6.5: 48.4: 6.5)\end{array}$ & $\begin{array}{c}3: 1: 5: 0 \\
(33.3: 11.1: 55.6: 0)\end{array}$ & 0.823 \\
\hline Increased respiratory support $^{\dagger}$ & $10(25.0)$ & $7(22.6)$ & $3(33.3)$ & 0.512 \\
\hline Lowest sodium (mmol/L) & $128.5(119-133)$ & $129.0(120-133)$ & $125.0(119-131)$ & 0.043 \\
\hline Highest potassium (mmol/L) & $5.9(4.7-9.0)$ & $5.8(4.7-7.7)$ & $6.1(5.4-9.0)$ & 0.276 \\
\hline $\operatorname{Cortisol}^{\ddagger}(\mu \mathrm{g} / \mathrm{dL})$ & $7.50(0.80-30.35)$ & $6.82(0.80-15.70)$ & $30.35(3.00-30.35)$ & 0.145 \\
\hline BUN (mg/dL) & $13(5-33)$ & $12(5-33)$ & $14(5-30)$ & 0.750 \\
\hline Creatinine (mg/dL) & $0.89(0.48-1.80)$ & $0.89(0.48-1.80)$ & $0.9(0.60-1.48)$ & 0.633 \\
\hline Inotrope use $\mathrm{e}^{\S}$ & $\begin{array}{c}3: 13: 12: 9: 3 \\
(7.5: 32.5: 30.0: 22.5: 7.5)\end{array}$ & $\begin{array}{c}2: 11: 9: 8: 1 \\
(6.5: 35.5: 29.0: 25.8: 3.2)\end{array}$ & $\begin{array}{c}1: 2: 3: 1: 2 \\
(11.1: 22.2: 33.3: 11.1: 22.2)\end{array}$ & 0.325 \\
\hline HCS use & $38(95.0)$ & $29(93.5)$ & $9(100.0)$ & 0.434 \\
\hline Time to HCS administration from hypotension (hr) & $13.5(1-178)$ & $14.0(1-178)$ & $13.0(2-139)$ & 0.919 \\
\hline HCS treatment duration (d) & $10(4-66)$ & $9(4-66)$ & $12(4-50)$ & 0.787 \\
\hline
\end{tabular}

Values are expressed as median (range) or number (\%).

*Room air: $\mathrm{O}_{2}$ prong: noninvasive ventilator includes high-flow nasal cannula: invasive ventilator; ${ }^{\dagger}$ Whether the need for ventilator mode was increased compared with before the late-onset circulatory collapse period; ${ }^{*}$ The serum cortisol level was measured just before hydrocortisone treatment was started. We did not obtain the value in four infants (three in the normal group and one in the brain lesion group); ${ }^{\circledR}$ None: dopamine: add dobutamine: add epinephrine: add norepinephrine.

Abbreviations: MBP, mean blood pressure; BUN, blood urea nitrogen; HCS, hydrocortisone. 
preterm infants with LCC to demonstrate the overall clinical characteristics of LCC and to discover the clinical factors that cause brain damage in LCC. To our knowledge, this is the first casecontrol study to investigate the risk factors for brain damage in preterm infants with LCC. Our results showed that older PNA, lower MBP, and lower serum sodium concentrations during the LCC period increased the incidence of brain damage in preterm infants with LCC.

LCC is defined as refractory hypotension that suddenly occurs in stable preterm infants $>1$ week after birth. The prevalence of LCC reported in a Japanese review was $6.3 \%$ in very low birth weight infants ${ }^{3)}$ and that in our study was $6.0 \%$ in infants aged $<35$ weeks GA. The incidence of LCC was high in relatively younger GA infants (25 to 26 weeks); however, most LCC events occurred at a relatively later PMA (30 to 34 weeks) in our study. In most cases, we observed abrupt LCC onset in clinically stable

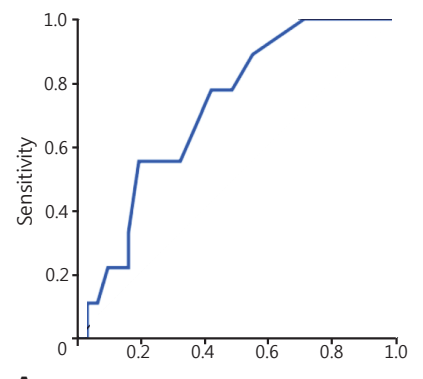

A
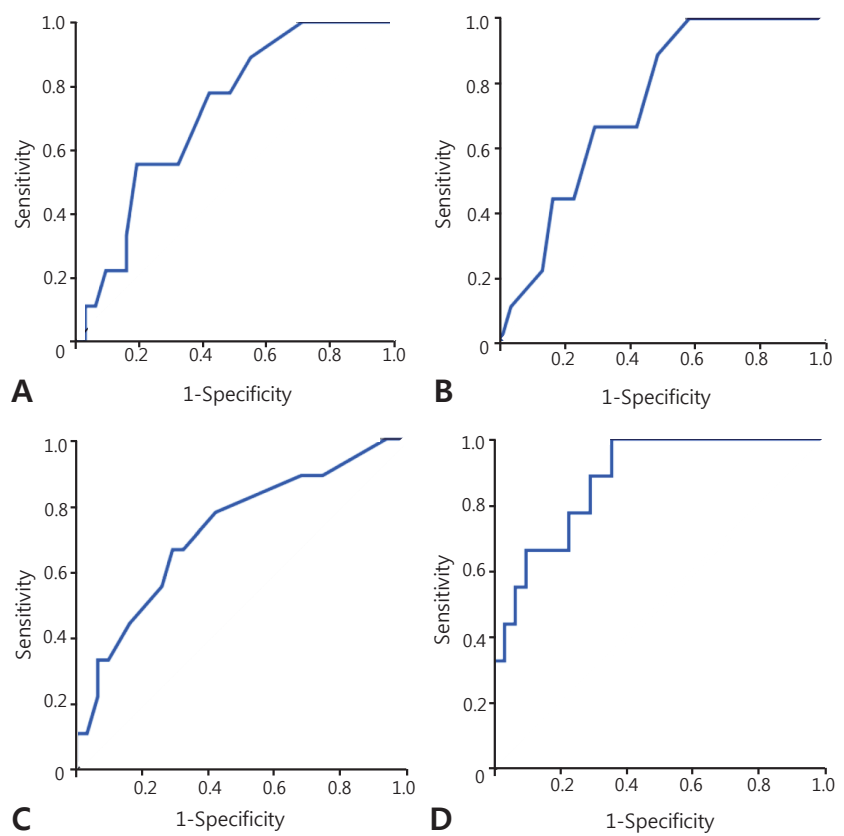

Figure 3 . Receiver operating characteristic (ROC) curves for predictors of brain damage in patients with late-onset circulatory collapse (LCC). (A) ROC curve of postnatal age as a predictor of brain damage. The area under the ROC curve was 0.720 (95\% confidence interval $[\mathrm{CI}], 0.552$ to $0.889 ; P=0.046$ ). (B) ROC curve of the lowest mean blood pressure as a predictor of brain damage. The area under the ROC curve was 0.740 (95\% CI, 0.581 to $0.899 ; P=0.030$ ). (C) ROC curve of the lowest serum sodium as a predictor of brain damage. The area under the ROC curve was 0.724 (95\% CI, 0.531 to $0.917 ; P=0.043$ ). (D) ROC curve of the predicted probability of logistic regression model for brain damage in infants with LCC. The area under the ROC curve was 0.882 (95 $\% \mathrm{CI}, 0.771$ to $0.992 ; P=0.001$ ). nonintubated infants with full enteral feeding. The brain volume increased to about $15 \mathrm{~mL} /$ week between 29 and 41 weeks of gestation, and about $65 \%$ of the brain volume of term infants was formed at 34 weeks. Because most of the brain formation process occurs at a later GA, the brain is more likely to sustain damage in the later preterm period ${ }^{12)}$. The median PMA of infants with LCC was 32 weeks, and the LCC onset time in all patients with brain damage was at 30 to 34 weeks PMA in our study. In this period, brain formation is more rapid, and the brain seems to be more vulnerable to the development of lesions.

In our study, PNA, MBP, and sodium levels were found to significantly contribute to brain lesion development in infants with LCC. The reason why older PNA affected brain damage in infants with LCC is unclear. LCC occurs mainly in the later preterm period, when most infants have relatively stable vital signs. When this stable period is prolonged, less frequent monitoring of blood pressure, input/output, and blood sampling is performed, which can delay the detection of symptoms and signs of LCC, as well as the treatment initiation. Therefore, even in long-term stable preterm infants, vital signs and general status should be carefully monitored for early detection of LCC.

The median MBP in the brain lesion group was $19 \mathrm{~mm} \mathrm{Hg}$, and the cutoff value for brain damage was $20.5 \mathrm{~mm} \mathrm{Hg}$. This is consistent with a previous study reporting an MBP threshold of ischemic cerebral blood flow in neonates of $<20 \mathrm{~mm} \mathrm{Hg}^{13)}$. A cerebral autoregulation mechanism exists to maintain a constant cerebral blood flow even when the arterial blood pressure changes. This process avoids damage to the brain in case of systemic hypertension or hypotension. In preterm infants, systemic blood pressure fluctuates widely, and autoregulation is more important. Therefore, hypotension could lead to decreased cerebral perfusion among preterm infants with impaired cerebral autoregulation and may result in the development of PVL ${ }^{14,15)}$.

In the presence of adrenal insufficiency, the secretion of antidiuretic hormone $(\mathrm{ADH})$ increases, resulting in water retention and a reduction in plasma sodium concentration ${ }^{16,17)}$. In the presence of cortisol deficiency, systemic blood pressure and cardiac output decrease, resulting in hypersecretion of $\mathrm{ADH}$, and a more important mechanism may be that $\mathrm{ADH}$ is an adrenocorticotropic hormone secretagogue ${ }^{18-21)}$. In our study, the median value of the lowest sodium level in infants with LCC was $128.5 \mathrm{mmol} / \mathrm{L}$, which significantly decreased to $125 \mathrm{mmol} / \mathrm{L}$ in the brain lesion group. Brain osmolality is in equilibrium with extracellular fluid osmolality. When hyponatremia occurs, plasma osmolality 
decreases and water moves to the brain according to osmotic gradients, resulting in cerebral edema. Brain edema also deteriorates cerebral perfusion ${ }^{22-24)}$. Moreover, rapid correction of hyponatremia causes an inverse osmotic gradient with excessive loss of water from the cells, causing brain dehydration and demyelination of white matter (called osmotic demyelination syndrome), and can lead to irreversible brain damage ${ }^{25-27)}$. As both severe hyponatremia and its rapid correction may cause brain damage, meticulous care should be employed, including using a volume expander and correcting hyponatremia at an appropriate rate, in patients suspected to have LCC.

Severe hyponatremia and hypotension associated with brain damage are signs of LCC, and hydrocortisone administration is the only treatment. According to a previous study, PVL with LCC causes more severe brain lesions than does PVL without LCC, and its prognosis is worse. The reason for severe brain lesions in LCC is believed to be ischemia during the period of refractory hypotension, which persists even with the use of inotropes and volume expanders ${ }^{28)}$. Therefore, rapid initiation of hydrocortisone treatment after LCC onset may be crucial. In previous studies, when hydrocortisone was administered, blood pressure increased after 2 hours and the inotrope requirement decreased from 6 to 12 hours after administration ${ }^{29,30)}$. However, unexpectedly, there were no significant differences in terms of hydrocortisone use, initiation time, and treatment duration between the two groups in our study.

The underlying pathophysiology of LCC is considered to be relative adrenal insufficiency; however, the etiology remains unclear. The concept of LCC is not yet widely accepted because LCC can be diagnosed based on clinical criteria. Nevertheless, it is important to understand the clinical features of LCC because clinicians have encountered this disease entity in the clinical field. Our study was conducted at a single institution and has the limitation of including a small number of patients. Further large multicenter follow-up studies for hydrocortisone administration and well-established protocols for overall LCC management are needed.

In conclusion, infants with older PNA, lower serum sodium level, and lower MBP during the LCC period had a higher incidence of brain damage. Therefore, LCC-associated signs should be closely monitored even in long-term stable preterm infants. Moreover, management for preventing severe hyponatremia and hypotension are important to minimize the occurrence of brain damage in infants with LCC.

\section{CONFLICT OF INTEREST}

No potential conflict of interest relevant to this article was reported.

\section{ACKNOWLEDGMENTS}

We thank Sohee Oh for helping with the statistical analyses.

\section{REFERENCES}

1. Ng PC, Lee CH, Lam CW, Ma KC, Fok TF, Chan IH, et al. Tran sient adrenocortical insufficiency of prematurity and systemic hypotension in very low birthweight infants. Arch Dis Child Fetal Neonatal Ed 2004;89:F119-26.

2. Shimokaze T, Akaba K, Saito E. Late-onset glucocorticoidresponsive circulatory collapse in preterm infants: clinical characteristics of 14 patients. Tohoku J Exp Med 2015;235:241-8.

3. Kawai M. Late-onset circulatory collapse of prematurity. Pediatr Int 2017;59:391-6.

4. Washio Y, Uchiyama A, Nakanishi H, Totsu S, Masumoto K, Kusuda S. Hemodynamic analysis in infants with late-onset circulatory collapse. Pediatr Int 2013;55:582-8.

5. Young RS, Hernandez MJ, Yagel SK. Selective reduction of blood flow to white matter during hypotension in newborn dogs: a possible mechanism of periventricular leukomalacia. Ann Neurol 1982;12:445-8.

6. Shin SM, Chai JW. Brain ultrasonographic findings of late-onset circulatory dysfunction due to adrenal insufficiency in preterm infants. Ultrasonography 2016;35:258-64.

7. Nakanishi H, Yamanaka S, Koriyama T, Shishida N, Miyagi N, Kim TJ, et al. Clinical characterization and long-term prognosis of neurological development in preterm infants with late-onset circulatory collapse. J Perinatol 2010;30:751-6.

8. Fernandez E, Schrader R, Watterberg K. Prevalence of low cortisol values in term and near-term infants with vasopressor-resistant hypotension. J Perinatol 2005;25:114-8.

9. Kluckow M. Low systemic blood flow and pathophysiology of the preterm transitional circulation. Early Hum Dev 2005;81: 429-37.

10. Munro MJ, Walker AM, Barfield CP. Hypotensive extremely low birth weight infants have reduced cerebral blood flow. Pediatrics 2004;114:1591-6.

11. Kobayashi S, Fujimoto S, Koyama N, Fukuda S, Iwaki T, Tanaka $\mathrm{T}$, et al. Late-onset circulatory dysfunction of premature infants 
and late-onset periventricular leukomalacia. Pediatr Int 2008; 50:225-31

12. Kinney HC. The near-term (late preterm) human brain and risk for periventricular leukomalacia: a review. Semin Perinatol 2006; 30:81-8.

13. Victor S, Marson AG, Appleton RE, Beirne M, Weindling AM. Relationship between blood pressure, cerebral electrical activity, cerebral fractional oxygen extraction, and peripheral blood flow in very low birth weight newborn infants. Pediatr Res 2006;59: 314-9.

14. Tsuji M, Saul JP, du Plessis A, Eichenwald E, Sobh J, Crocker R, et al. Cerebral intravascular oxygenation correlates with mean arterial pressure in critically ill premature infants. Pediatrics 2000;106:625-32.

15. Greisen G. Autoregulation of cerebral blood flow in newborn babies. Early Hum Dev 2005;81:423-8.

16. Oelkers W. Hyponatremia and inappropriate secretion of vasopressin (antidiuretic hormone) in patients with hypopituitarism. N Engl J Med 1989;321:492-6.

17. Ishikawa S, Schrier RW. Effect of arginine vasopressin antagonist on renal water excretion in glucocorticoid and mineralocorticoid deficient rats. Kidney Int 1982;22:587-93.

18. Wolfson B, Manning RW, Davis LG, Arentzen R, Baldino F Jr. Colocalization of corticotropin releasing factor and vasopressin mRNA in neurones after adrenalectomy. Nature 1985;315:59-61.

19. Kalogeras KT, Nieman LK, Friedman TC, Doppman JL, Cutler GB Jr, Chrousos GP, et al. Inferior petrosal sinus sampling in healthy subjects reveals a unilateral corticotropin-releasing hormone-induced arginine vasopressin release associated with ipsilateral adrenocorticotropin secretion. J Clin Invest 1996;97: 2045-50.

20. Papanek PE, Raff H. Physiological increases in cortisol inhibit basal vasopressin release in conscious dogs. Am J Physiol 1994; 266(6 Pt 2):R1744-51.

21. Schrier RW. Body water homeostasis: clinical disorders of urinary dilution and concentration. J Am Soc Nephrol 2006;17: 1820- 32.

22. Adrogue HJ, Madias NE. Hyponatremia. N Engl J Med 2000;342: 1581-9.

23. Sjoblom E, Hojer J, Ludwigs U, Pirskanen R. Fatal hyponatraemic brain oedema due to common gastroenteritis with accidental water intoxication. Intensive Care Med 1997;23:348-50.

24. Ayus JC, Armstrong D, Arieff AI. Hyponatremia with hypoxia: effects on brain adaptation, perfusion, and histology in rodents. Kidney Int 2006;69:1319-25.

25. Ayus JC, Achinger SG, Arieff A. Brain cell volume regulation in hyponatremia: role of sex, age, vasopressin, and hypoxia. Am J Physiol Renal Physiol 2008;295:F619-24.

26. Upadhyay A, Jaber BL, Madias NE. Incidence and prevalence of hyponatremia. Am J Med 2006;119(7 Suppl 1):S30-5.

27. Giuliani C, Peri A. Effects of hyponatremia on the brain. J Clin Med 2014;3:1163-77.

28. Kobayashi S, Fujimoto S, Fukuda S, Hattori A, Iwaki T, Koyama $\mathrm{N}$, et al. Periventricular leukomalacia with late-onset circulatory dysfunction of premature infants: correlation with severity of magnetic resonance imaging findings and neurological outcomes. Tohoku J Exp Med 2006;210:333-9.

29. Noori S, Friedlich P, Wong P, Ebrahimi M, Siassi B, Seri I. Hemodynamic changes after low-dosage hydrocortisone administration in vasopressor-treated preterm and term neonates. Pediatrics 2006;118:1456-66.

30. Seri I, Tan R, Evans J. Cardiovascular effects of hydrocortisone in preterm infants with pressor-resistant hypotension. Pediatrics 2001;107:1070-4. 